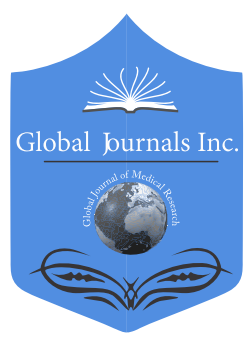

GLOBAL JOURNAL OF MEDICAL RESEARCH: K

INTERDISCIPLINARY

Volume 20 Issue 14 Version 1.0 Year 2020

Type: Double Blind Peer Reviewed International Research Journal

Publisher: Global Journals

Online ISSN: 2249-4618 \& Print ISSN: 0975-5888

\title{
An Overview of the Immune Dysregulation in COVID-19 with Immunization Priority in the Indian Context
}

By Aditi Munmun Sengupta, Diptendu Chatterjee,
Salil Kumar Bhattacharya \& Rima Ghosh

University of Calcutta

Abstract- The review was aimed at understanding the immune dysregulation by the novel virus, COVID-19. The review also highlighted the major findings that have been published in the previous studies through secondary research about the mechanism of immune dysregulation, transmission and progression of the virus across the globe. Adults above the age of 60 years and with co-morbidities such as cardiac and renal dysfunction, hypertension and diabetes are predisposed to greater risk of COVID-19 infection. The virus impairs the physiological, psychological and metabolic functioning of the patient. The paper gained an insight into the need for immunization priority for the Indian population and recommendations for the effective policy making in vaccine development and distribution.

Keywords: Covid-19, transmission, immune dysregulation, Covid-19 vaccine, immunization, immune dysregulation.

GJMR-K Classification: NLMC Code: WD 308

Strictly as per the compliance and regulations of:

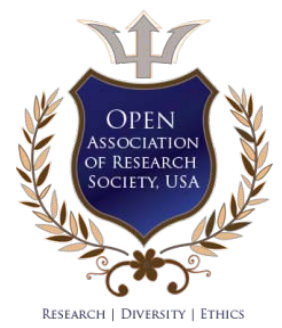

(C) 2020. Aditi Munmun Sengupta, Diptendu Chatterjee, Salil Kumar Bhattacharya \& Rima Ghosh. This is a research/review paper, distributed under the terms of the Creative Commons Attribution-Noncommercial 3.0 Unported License http://creativecommons.org/licenses/by-nc/3.0/), permitting all non-commercial use, distribution, and reproduction in any medium, provided the original work is properly cited. 


\title{
An Overview of the Immune Dysregulation in COVID-19 with Immunization Priority in the Indian Context
}

\author{
Aditi Munmun Sengupta ${ }^{\alpha}$, Diptendu Chatterjee ${ }^{\sigma}$, Salil Kumar Bhattacharya ${ }^{\circ}$ \& Rima Ghosh $^{\omega}$
}

Abstract- The review was aimed at understanding the immune dysregulation by the novel virus, COVID-19. The review also highlighted the major findings that have been published in the previous studies through secondary research about the mechanism of immune dysregulation, transmission and progression of the virus across the globe. Adults above the age of 60 years and with co-morbidities such as cardiac and renal dysfunction, hypertension and diabetes are predisposed to greater risk of COVID-19 infection. The virus impairs the physiological, psychological and metabolic functioning of the patient. The paper gained an insight into the need for immunization priority for the Indian population and recommendations for the effective policy making in vaccine development and distribution.

Keywords: Covid-19, transmission, immune dysregulation, Covid-19 vaccine, immunization, immune dysregulation.

\section{Graphical Abstract}

\section{Mechanis m of infection of SARS-CoV-2}

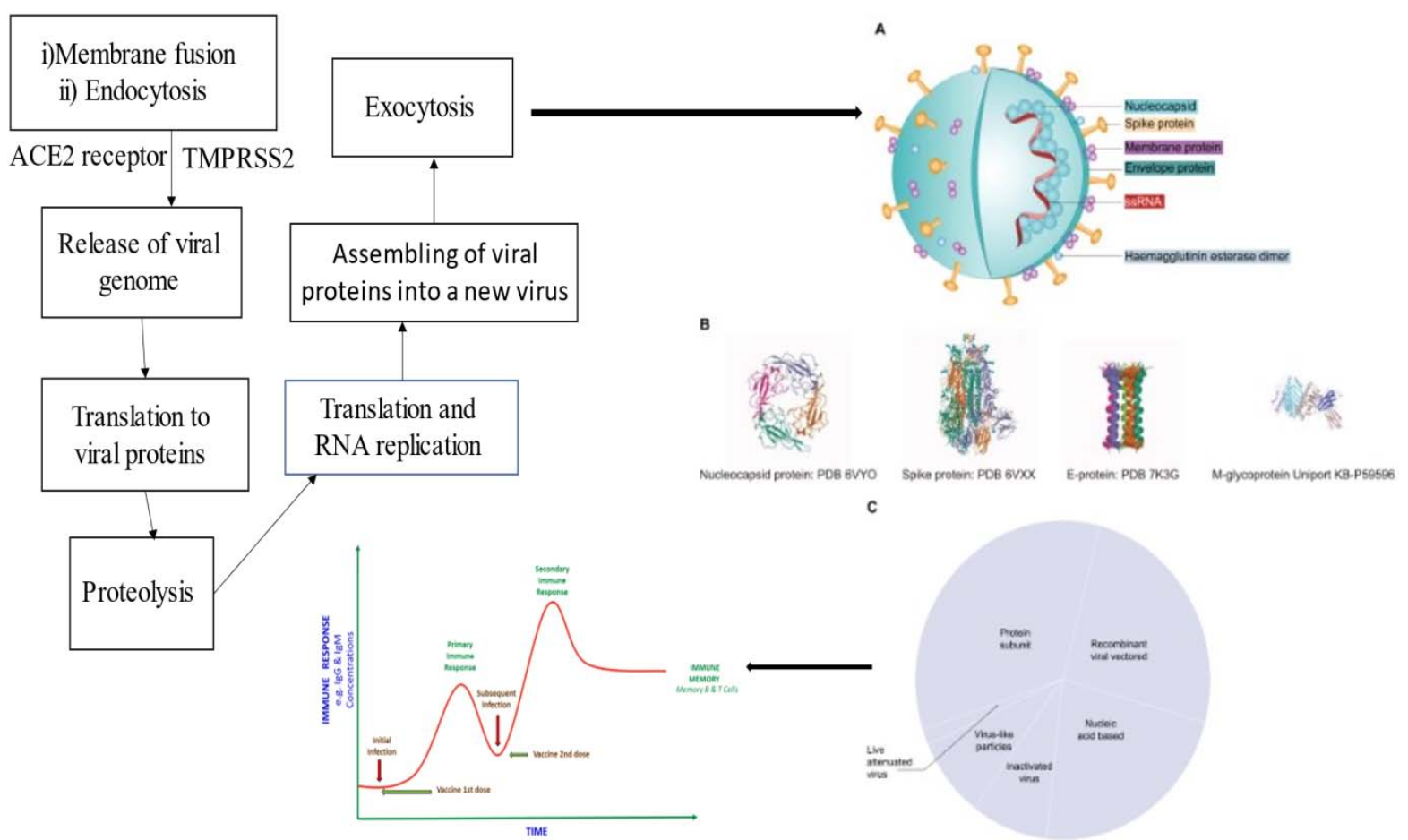

Corresponding Author a: Medical Officer, CK Birla Hospitals; Research Scholar, University of Calcutta; Harvard Medical School, Post Graduate Association Member, Kolkata, India.e-mail: sengupta2aditi@gmail.com

Author б: Assistant Professor (Stage-3), Dept. of Anthropology and Deputy Registrar of University of Calcutta, Kolkata, India.

Author p: Ex-Professor Dept. of Community Medicine, Calcutta Medical College, Kolkata, India.

Author w: Junior Research Fellow (UGC-NET), University of Calcutta, Kolkata, India. 


\section{Types of COVID-19 Vaccines}

\author{
Active Immunity (Vaccination) \\ - DNA Vaccine(Inovio ) \\ - RNA Vaccine(Moderna, Pfizer) \\ - Viral Vector(Oxford,Astrazeneca,Janssen,Gamalaya - \\ Sputnik,CanSino Biologics) \\ - Viral Subunit (Novavax,Adaptvac,Clover Biopharma) \\ - Live Attenuated(Codagenix,Indian Immunologicals Ltd.) \\ - Inactivated Virus (Sino Vac, SinoParm) \\ - VPL(Virus Like Particles) \\ - Split Virus Vaccines (eg. Flu Vaccines) \\ - RNP(Ribonucleoprotein) Vaccine.
}

Passive Immunity (Antibody Administration)

\section{- Antibodies}

Monoclonal Antibodies( e.g.Bamlanivimab )

Polyclonal Antibodies( e.g.Regeneron)

- Convalescent Plasma

- mRNA induced Antibody

\section{INTRODUCTION}

( ntil the outbreak of Corona virus (CoV), this family of viruses was greatly overlooked. With the outbreak of SARS, these viruses are being studied in elaborate propelling the research for vaccines. After the emergence of mysterious cases of pneumonia detected in the Wuhan city (China), this causative agent transmitted extensively to 210 countries and across territories. According to the World Health Organization (WHO), this disease is referred to as Corona virus Disease-2019 (COVID-19) suspected to have its origin from Huanan seafood market. Ji et al., (2020) made the first claim and identified snakes as COVID-19 host and then many claims were made until experts collectively concluded that bats harbor the virus (Gorbalenya et al., 2020). The main mode of COVID-19 transmission is human-human via droplets generated during sneezing, coughing or talking inhaled by a healthy individual (Keni et al., (2020). Asymptomatic carriers are super infectors with no sign or mild symptoms of the disease posing challenges for the Indian Government on how to identify them as they could account for $80 \%$ of the infections (Keni et al., 2020).

Apart from cytopathic effects witnessed in nonsurvival COVID-19 cases, immune dysregulation substantially contributes to pathogenesis. Immunomodulatory agents like glucocorticoids, interleukin-6 blockers have been observed to be beneficial for the treatment. This virus is associated with reduction in number and function of the CD8+ T-cells

\section{Vaccine availability and Eligible groups}
a)Health care workers and first responders b)Patients with one or more comorbidities c)Patients over 65 years with no comorbidities d)Over 65 years in congregate settings e)Essential workers not fitting other categories
f) Street residents and homeless

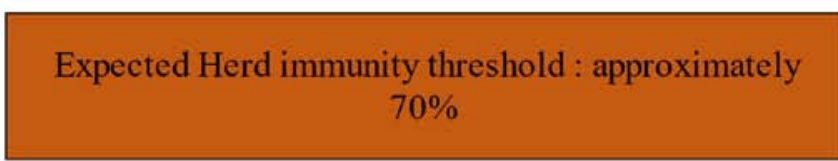

and NK-cells (Natural Killer) leading to immune dysregulation and delayed viral clearance (Praveen et al., 2020). The combination of antiviral drugs like remdesivir, IFN therapy and hydroxychloroquine combined with immunomodulatory therapy in hyperinflammatory phase can be considered a suitable approach in COVID-19 management. The development of a vaccine with use of mRNA-based approach can be a more viable option for producing immunity against COVID-19 including isolated spike virus proteins and live attenuated virus.

As recognized by $\mathrm{WHO}$, immunization through vaccination is the need of the hour and essential health service to be taken up by the countries for the prevention of COVID-19 (World Health Organization, 2020). Scientists around the world are joining hands to develop vaccines to impede the progression of this overwhelming pandemic. Moderna and the Vaccine Research Centre is working on mRNA based vaccine encapsulated in lipid nanoparticles whereas Serum Institute of India in collaboration with Codagenix is currently focused on development of live attenuated vaccine. Biotech ventures in India are working together to develop subunit vaccines, inactivated viral vaccines, attenuated recombinant vaccines and using codonoptimization. Therefore, the following review paper will gain insights into the emergence, transmission, immune dysregulation concerning public health, immune response of the individuals, and overview of vaccine development and understanding of COVID-19 vaccine. 


\section{Literature RevieW}

a) Impact of COVID-19 regarding the health concern

For the past 30 years, novel coronavirus is a once-per-decade that has pushed health infrastructure to limits posing a serious health concern. Transmissibility and severity of the disease are the two critical factors that would determine the public health impact of the pandemic. The virus has high transmission rate and quite severe case fatality rates (CFR) is greater being a potential health threat (Binns, Low \& Kyung, 2020). The pandemic has affected millions of lives making them sick or being killed due to disease spread. One out of every six individuals gets affected by the virus and develops difficulty in breathing. They can also develop pneumonia and breathing complications requiring urgent medical admission. COVID-19 majorly affects the respiratory system causing shortness of breath and coughing. People above the age of 60 years are at high risk of severe infection. The body's immune response to the infection includes fever, pneumonia, loss of taste and smell, sore throat, muscle pain and cough. It has a major impact on health where tiny sacs in lungs are filled with fluid causing breathing difficulty. People with serious heart conditions, type 2 diabetes, weakened immune system due to transplant or surgery, kidney and respiratory diseases are at higher risk of being infected. The incubation time is $2-14$ days during which the suspected patients remain in quarantine. Patients suffer from acute respiratory distress syndrome (ARDS) that requires ventilation and it has been observed that mortality rate becomes high within two days of admission (Zheng et al., 2020).

The main cause of death is the inflammation of lungs discussed above that serves as an entry point for infections associated with secondary infections, coagulopathy and end-organ failure (Shi et al., 2020). The age-dependent defects like excess type 2 cytokines production in the B-cell and T-cell may lead to delayed pro-inflammatory responses and deficiency in viral replication control leading to poor health outcomes. In addition, bacterial infections could also cause sepsis syndrome reported in $40 \%$ of community-acquired pneumonia because of viral infection (Chaubey, 2020). About 58,064,186 are infected cases with a mortality rate of 1,380,548 worldwide. In India, out of 90,54,528 confirmed cases, 1,32,797 are deceased cases with a recovery rate of $84,80,299$ (Worldometer, 2020). The deaths are occurring among people aged above 60 years and with underlying comorbidities. COVID-19 is resulting in severe disease posing a public health system including admission to intensive care units, hospitalization and death. The cases are doubling every 5-10 days challenging the health system and disrupting the socio economic condition of India.

$80 \%$ of COVID-19 cases are asymptomatic or mild with $15 \%$ and $5 \%$ critical infections requiring oxygen and ventilation ensuring case isolation. The increased severity and mortality of the disease includes individuals above the age of 55 years with multiple co-morbidities, hypertension, obesity, immune-suppression and hypoxia. This predisposes patients to unfavorable treatment with increased risk for in tubing and death (Verity et al., 2020). In a paper published by Gallo Marin et al., (2020) the disease severity includes coagulation defects, cardiac dysfunctions, alterations in WBC blood cell counts, liver injury and renal dysfunction. Largevessel stroke is one of the major health concerns that have been witnessed in severe COVID-19 cases even in younger patients. 12 out of 67 patients died on admission due to elevated D-dimer levels, developed hypercoagulability, delayed prothrombin time and thrombocytopenia (Zhang et al., 2020). Liu et al., (2020) studied that severe fulminant myocarditis and systolic dysfunction were reported in COVID-19 patients. The patients who received ICU care were more likely to suffer from acute cardiac injury as compared to non-ICU patients with elevated troponin levels being an independent risk factor for mortality.

Another health concern in COVID-19 patients is WBC count alterations along with lower granulocyte counts linked to severity of the disease. Huang et al., (2020) conducted a meta-analysis and identified that significant reductions in lymphocyte counts, B cells, CD8+ T cells and CD4+ cells and NK cells is associated with severe COVID-19 cases as compared to mild or moderate cases. Huang et al., (2020) highlighted that in critical COVID-19 patients, elevations in level of alanine aminotransferase (ALT) and aspartate aminotransferase (AST) are more likely to occur and end-organ damage. The need for mechanical ventilation increases for patients with hypoalbuminemia, abnormal liver enzyme levels also being an independent predictor of COVID-19 mortality. Kidney dysfunction and failure has been reported in severe COVID-19 cases (Guan et al., 2020). Systemic inflammatory and vascular complications may be indicative of renal failure strongly suggesting that this virus has the capacity to infect the tubular renal epithelium. Therefore, to improve health outcomes in COVID-19 patients, it is vital to identify and validate the predicting factors in COVID-19 progression.

\section{b) Overview of the immune response concerning COVID-19 infection}

As COVID-19 virus in severe patients mainly spreads to the respiratory tract with cytokine storms and lymphopenia as accompanying agents, the early recognition of immunological phenotypes can be helpful in prompt identification of patients who would progress to severity. The virus has a crown-like single stranded RNA having a ridge on the concave surface. It has a large binding interface that makes more contact with the $\mathrm{N}$-terminal of Angiotensin-Converting Enzyme 2(ACE2) with greater affinity (Ong et al., 2020). The virus is 
transmitted through droplets from sneezing and coughing entering the nasal system through inhalation. It then gradually starts replicating where ACE2 acts as the main receptor. The spike (S protein) on the surface of the virus enters inside the host cell and binds to the ACE2 receptor. The enzyme, furin present in the host cell acts as the main factor for the virus entry into the host. The virus starts to propagate with the help of the host's innate immune response and as such is detected in nasal swabs. Then, it reaches the respiratory tract when there is clinical manifestation of the disease as cytokine (innate response), may be a predictor of the clinical course (Chowdhury et al., 2020). For 80\% Individuals with mild or moderate symptoms has virus infection mainly restricted to conducting and upper airways that can be managed at home. However, rest $20 \%$ of them develops severe cases due to pulmonary infiltrates leading to high mortality rates. Once COVID-19 virus reaches the alveolar and bronchiolar spaces, it targets the bronchial epithelium cells and type-II ACE2+ pneumocytes in alveolar epithelium. It induces autophagy, basal membrane detachment and ACE2 expression inhibition. Hence, it allows angiotension II binding to the AT1aR receptor resulting in acute damage of lungs. Here, the infected cells show early defense mechanisms through production of IFN (type-III and I) activating inhibition. Later, virions release reaches the neighboring cells resulting in systemic infection by ACE2 + widely distributed in tissues (Azkur et al., 2020).

When our body detects a novel pathogen or virus like COVID-19, the defensive cells in innate immunity release cytokines that alerts the other cells involved in immunity. After the virus entry into the host cell, the Pattern Recognition Receptors (PRR), TLR7 and TLR8 are expressed by the epithelial and local cells of the innate immunity like alveolar macrophages. PRRs after ligand binding, recruits adaptor proteins for the activation of down-stream transcription factors like NF$\kappa \mathrm{B}$, interferon regulatory factor (IRF) and $\mathrm{AP}-1$ for the production of chemokines and antiviral Interferons (Type-I and -III) (Mortaz et al., 2020). The innate response cells like monocytes, dendritic cells (DCs) NK cells and polymorphonuclear leukocytes that in turn produce more chemokines like MCP-1, IP-10 and MIG that are capable of lymphocytes recruitment. This, in turn, makes DCs recognize the COVID-19 virus antigens. This is supported by the fact that viruses infect pneumocytes (type-I and type-II), alveolar macrophages depicting better capacity of replication in pulmonary tissues supported by elevated serum levels in positive patients (Prompetchara, Ketloy \& Palaga, 2020).

The transition from innate to adaptive responses is quite critical for the infection progression. Although poorly understood, the immune regulatory cells may develop either exacerbated or protected inflammatory response. The protective response is provided by $T$ cell dependent cells with CD4 aided B cells binding geared towards neutralizing antibodies production and elimination of infected cells by cytotoxic CD8 cells. In $80 \%$ of cases, CD8 acts as infiltrating cells. The exacerbated response is unable to inhibit and eliminate the viral replication in the infected cells leading to cytokine storm and disseminated intravascular coagulation peak at day 14 up to day 28 (GiamarellosBourboulis et al., 2020).

NK cells and Cytotoxic lymphocytes (CTLs) are also important for the viral infection control and exhaustion of functional cytotoxic lymphocytosis that may increase the severity of the disease. The cell numbers and function decrease with up regulation of NK inhibitory receptor CD94/NK group 2 member A (NKG2A) upregulation in COVID-19 comprising immune response. There is an increase in $T$ cell apoptosis with decreased CD4+ and CD8+ T cells in the COVID-19 patient's peripheral blood. In addition, these cells have impaired activation with the appearance of CD25, CD28, and CD69 on the subsets of T cells. This fact supports the prolonged clearance of virus and delayed adaptive immune response in severe patients (Rao et al., 2020).

The Indians could have intrinsic immunity to resist the COVID-19 infection as compared to other countries across the globe. India has lowest test positivity rates with $3.3 \%$ critical or severe cases with less mortality rates are remarkable and quite encouraging. Firstly, the immunity of Indian population is broad due to exposure to a wide range of pathogens and microbial load that could specifically make them immunologically strong and mechanisms of crossreactive memory $T$ cells that need further exploration (Chinnaswamy, 2020). Further, BCG vaccination usage in India may have provided innate and adaptive immunity against the pathogens like COVID-19. Indians had begun to react aggressively to this pandemic where Indians have a lower number of deaths as compared to other countries. Trained innate immunity is a new paradigm like adaptive immunity arm that could have some memory to fight against this sort of encounter. The innate immune cells like NK, DCs and macrophages get trained due to first encounter with similar stimulus and retain the training as memory in epigenetic landscape. The Indian hosts have been exposed to endemic intracellular pathogens like protozoan or $M$. tuberculosis. This training of innate immunity and Indian host exposure to a myriad of viral infections could be helpful in protecting the majority if Indian population from COVID-19 (Singh, Maurya \& Singh, 2020).

\section{c) Immune dysregulation in COVID-19 Infection}

COVID-19 affects multisystem and exhibits an array of health issues, therefore, understanding of metabolic, physiological or psychological consequences in the immune system is important for better health outcomes. For a COVID-19 patient, metabolism undergoes changes at all levels of 
organization and adaptive responses by the immune host. Poor metabolic health with abnormalities like cardiovascular abnormalities or hyperlipidaemia can be detected in COVID-19 patients (He et al., 2020). There might be disruptions in lysophosphatidylinositol (LPI) that could in turn hamper glucose homeostasis suggesting abnormal insulin release and metabolism. Physiological changes like damage to metabolic and endocrine organs like liver, pancreas may contribute to further metabolic syndrome in COVID-19 patients. Hyperglycemic condition lowers the immune response in COVID-19 patients that causes abnormality in the metabolism of the host. The immune profiles of patients depicted high levels of LDH, CTnl and pro-BNP indicating severe cardiac dysfunctioning and cardiac failure. Low levels of uric acid indicated potential dysfunctioning of renal or liver metabolism in severe patients. The exhaustion of $\mathrm{T}$ cells makes the patient potential towards other pathogens with chances of cross-contamination (Ayres, 2020).

COVID-19 also affects the physiological functioning in patients affected with the virus. Zhao et al., (2020) conducted a study on COVID-19 survivors to study the pulmonary functioning and physiological characteristics after three months of discharge. The results suggested that physiological and radiological abnormalities with impaired pulmonary functioning exist in survivors like abnormal carbon monoxide diffusion capacity (DLCO) because of D-dimer levels at the time of admission. Moreover, diffuse alveolar damage (DAD), pneumocyte activation, lymphocytic inflammation, hyaline membrane formation and proteinaceous edema are a wide range of respiratory abnormalities witnessed in positive patients. Diaphragm weakness due to mechanical ventilation in ICU patients demonstrated muscle thinning and reduced motion in critical patients (Brosnahan et al., 2020).

The psychological impact of COVID-19 is concerning as it has spread widely becoming pandemic. The strict lockdown, social restriction and isolation had a mental impact linked to feelings of uncertainty and frustration and individuals with preexisting mental problems are at high risk. The pandemic acted as a stressor for the patients that affected their mental health in response to the stressful event called as "coronaphobia" (Dubey et al., 2020). High levels of anxiety, perceived stress and Post Traumatic Stress Disorder (PTSD) presented among the patients in response to the pandemic. COVID-19 recovered patients showed signs of anxiety, confusion and insomnia that make them prone to compulsive disorder and depression symptoms. The virus has serious implications on one's well-being and they are likely to experience long-term mental issues. As discussed above, ACE2 malfunctioning is associated with covid-19 propagation and transmission. As it is widely spread in tissues and present in the brain and blood-brain barrier, the virus may target the ACE2 receptors and cause central nervous system (CNS) infections.

\section{d) Basic principles of immunization in COVID-19 infection}

A variety of COVID-19 vaccines are being developed around the world. All of them share one thing in common: they all stimulate a primary immune response so that body can develop memory $\mathrm{B}$ and $\mathrm{T}$ cells against the SARS-CoV-2 virus. The development of immune memory by vaccines is what will protect the person against subsequent COVID-19 infection.

Depending on how many times the body is exposed to the virus or vaccinated, the body can generate two types of immune responses.

The body generates a primary immune response when exposed to SARS-CoV-2 virus for the first time or gets the $1^{\text {st }}$ dose of the vaccine. The primary immune response is slow and weak as it takes days for the body to generate enough antibodies and $T$ cells to eliminate the virus. However, the body generate enough antibodies and T-cells that 'remember' the SARS-CoV-2 virus, generating immune memory.

When the virus enters the body for the second time or the $2^{\text {nd }}$ dose of the vaccine is given, the body develop a secondary immune response. The secondary immune response is stronger and quicker than the primary immune response as memory B and T cells are rapidly inactivated.

This results in higher antibody concentrations and T-cell counts around the body to eliminate the virus more quickly, reducing the symptoms and severity of COVID-19.In addition more memory B and T cells are produced after infection which strengthens the memory of the SARS-CoV-2 virus. The development of immune memory by vaccines is what will protect the individual against subsequent COVID-19 infection.

Each COVID-19 vaccine has distinct advantages and disadvantages, but the development of different COVID-19 vaccines provide some redundancy and overlap. In case a vaccine is unsafe in humans or fails to protect people against COVID-19, the world has other COVID-19 vaccines that it can trial and produce. It is this pursuit of multiple vaccines that will allow the global population to be immunized sooner, allowing the possibility to allow COVID-19 to be eliminated so that the world can start to recover from the pandemic (Speiser et al., 2020).

\section{e) Overview of vaccine development for COVID-19 infection}

In the current pandemic triggered by corona virus, immunization through vaccination of the population is recognized as a major priority in public health. Immunization in corona virus is of utmost priority where pandemic has hit the most. As no clinical data has suggested an effective candidate vaccine for corona virus, development of vaccines and 
immunization humans is the need of the hour and in curtailing the risk of spread of the disease. Extensive research is going on in vaccine development that would be effective for preventing the disease (Schwartz, 2020).

"Candidate" vaccines would be promising and the fastest process that may take weeks. More than 160 vaccine candidates are battling to combat the novel corona virus at different stages of clinical trials where 27 have reached human trial phase. Reports suggested that by the beginning of 2021, Oxford University's candidate vaccine 'ChAdOx1 nCoV-19' is a front runner currently undergoing the last stage of clinical trial, phase III of human trials in Brazil, UK and South Africa among 1600 volunteers dosed with it. Serum Institute of India (SII), Pune is conducting Phase II and III trials with Swedish-British firm AstraZeneca for producing vaccines for middle and low-income countries (Chen et al., 2020). mRNA-based vaccines have been a popular approach over conventional vaccines. SIl has also tied up with Codagenix, a US-based biotech firm for the development of a live-attenuated vaccine. It also tied up with Themis Bioscience, Austrian Biotech Company to deploy measles virus as a vector for injecting antigen or corona virus protein.

Biontech and Pfizer is concluding phase III candidate vaccine, BNT162b2 that ensures to meet the entire primary efficacy among adults over the age of 65 years. It showed 95\% primary efficacy against the virus beginning 28 days after the first dosage, 170 confirmed cases and 162 in placebo group versus vaccine group having 8 . They have achieved FDA milestones of safety data and are well tolerated among the participants with no safety issues. Pfizer is confident to provide 50 million vaccines by the end of year 2020 (Le et al., 2020).

Russia is developing Liposome-encapsulated DNA-protein COVID-19 Spike antigens vaccine and DNA coding sequence based on HSB antigens called Sputnik V. Moderna's mRNA-1273 is the first vaccine going for human trials having promising prospects like mRNA sequence encoding for $S$ protein (Burki, 2020). After phase I, the vaccine produced a mean antibody tier that is neutralizing in elderly subjects with a safety profile. Therefore, immunization through vaccination can be helpful in putting an end to the current pandemic situation.

\section{f) Herd immunity and COVID-19 vaccine in India}

The guidelines for vaccination in India is under development by Ministry of Health, Government of India. It is expected that the vaccine for the novel virus SARSCoV-2 will cost 1000 INR per dose. In 2020, the estimated total population of India amounted to approximately 1.38 billion people. Achieving a target of $70 \%$ in herd immunity is expected to stop the transmission of the virus. Currently, there is insufficient confirmed information on reinfection to determine how that will influence the course of the pandemic. Vaccine allocation can be specifically targeted to highly exposed populations, such as health care workers and first responders, second priority can be given to the task force like the police and individuals who are susceptible and have possibilities of frequent contacts. They may have a significantly greater impact on reducing viral circulation than naturally acquired immunity specifically if the acquired protective immunity requires boost through reinfection.(Dhot PM et al.,2020)

A measure of the infection levels in the population has been provided through the sero-surveys conducted by the Indian Council of Medical Research (ICMR). The study done between August 17 and September 22, 2020 found the prevalence at $15.6 \%$ of the population in urban slums and $8.2 \%$ in non slum areas. In Delhi, a seroprevalence of $29.1 \%$ was found in the second round estimates. The study revealed that for every reported COVID-19 case, there were 26-32 infections, down from 81-130 infections per reported case in May. In Mumbai, an independent study estimated around $45 \%$ prevalence in slums and $18 \%$ seroprevalence in non slums in the second round (ICMR Serosurvey.,2020; Serology Survey.,2020)).

It can be predicted that the susceptibility of a considerable section of people, who are still unexposed to SARS-CoV-2 does exist and the risk of urban slums is twice that in non slum areas; and almost 4 times higher than the risk in rural settings. Therefore, in the absence of a reliable vaccine, the vast majority of people everywhere irrespective of waxing and waning in daily caseloads continue to be vulnerable.

\section{ili. ReseARCH GAP}

The overview discussed the serious implications of COVID-19 on the metabolic, physiological and psychological well-being of the patients affected with the disease. Corona disrupts the functioning of the immune system that indicates immunization of the population and development of vaccines. The previous sections illustrated that there is a need for immunization priority among the individuals especially Indian population. Hence, this review provides an update on the immune dysregulation that can act as a basis for the vaccine development. In this present review paper, immunization priority has been highlighted in context to Indian population where the existing literature has helped in explaining the predisposing factors and reasons for immunization priority that further helps in filling the knowledge gap.

\section{Findings and Discussions}

This section summarizes the findings that are based on the analysis that has been made reviewing the existing literature. Since the outbreak of corona virus, the virus has affected 210 countries across the globe. It disrupts the immune system of an individual and makes 
them susceptible to other infections. People above the age of 60 years and with co-morbidities are prone to virus infection. The patient develops symptoms like fever, coughing, shortness of breath and loss of taste and smell. Individuals with co-morbidities like renal dysfunction, hyperglycemia, hypertension, cardiac issues make them predispose to the infection. The innate and adaptive immune cells and responses are involved in the viral infection. The pandemic has seriously challenged the health system of a country. Countries are working at breakneck speed to develop vaccines to curb the risk of infection. The paper highlighted the updates on vaccine development that are happening across the globe. However, the review paper helped to bridge the potential knowledge gap and tried to raise awareness about prioritizing the laboratory studies and scientific analysis of viral transmission and pathogenesis for immunization and vaccine development.

\section{Conclusion}

The review paper gave an insight into the trends of emergence, transmission and progression of the disease across populations. The immune dysregulation in COVID-19 makes the patient susceptible to other infections and co-morbidities acts as a risk factor for the progression of the disease. The pandemic has challenged the healthcare system in the context of vaccine development and its approval. India's journey to recovery from this pandemic could be possible by developing strategies for quickly producing, gaining access and effective delivery of vaccines for the population. There is a need to establish a task force for determining production, accessibility and distribution of covid-19 vaccine. A nationwide plan is required for the development and distribution of vaccines that balances efficiency, effectiveness and equity. Lastly, strong resourcing in resource and time intensive efforts would help the scientific community to respond to the virus quickly.

\section{ACKNOWLEDGEMENT}

We acknowledge heartfelt thanks to Scholars and Professors of University of Calcutta, and CK Birla Hospitals Doctors and Staffs for their continuous support and encouragement in this project. The authors sincerely acknowledge the support of Prof. BibhutiSaha, HOD, Dept. of Tropical Medicine for his encouragement and Dr. Kamalesh Sarkar, Director-NIOSH-ICMR for his able guidance. The authors also are grateful to Delvin Specialities for providing literature help.

Financial Assistance and Sponsorship:

Sri Sarosij Ray Memorial Research Support Fund

Conflict of interest:

We declare that we have no conflicts of interest.

\section{Contribution of authors:}

AMS- Conceptualized and designed the study, literature search, prepared first draft of the manuscript, critical revision of the manuscript; DC- Conceptualized the study, Interpretation, critical revision of the manuscript; SKB- Concept of the study, revision of the manuscript; RG- Literature search, review of the study

\section{References Références Referencias}

1. Ayres, J. S. (2020). A metabolic handbook for the COVID-19 pandemic. Nature metabolism, 2(7), 572-585.

2. Azkur, A. K., Akdis, M., Azkur, D., Sokolowska, M., van de Veen, W., Brüggen, M. C., ... \& Akdis, C. A. (2020). Immune response to SARS-CoV-2 and mechanisms of immunopathological changes in COVID-19. Allergy, 75(7), 1564-1581.

3. Binns, C., Low, W. Y., \& Kyung, L. M. (2020). The COVID-19 Pandemic: Public Health and Epidemiology. Asia-Pacific Journal of Public Health.

4. Brosnahan, S. B., Jonkman, A. H., Kugler, M. C., Munger, J. S., \& Kaufman, D. A. (2020). COVID-19 and Respiratory System Disorders: Current Knowledge, Future Clinical and Translational Research Questions. Arteriosclerosis, thrombosis, and vascular biology, 40(11), 2586-2597.

5. Burki, T. K. (2020). The Russian vaccine for COVID-19. The Lancet Respiratory Medicine, 8(11), e85-e86.

6. Chaubey, G. (2020). Coronavirus (SARS-CoV-2) and Mortality Rate in India: The Winning Edge. Frontiers in Public Health, 8, 397.

7. Chen, W. H., Strych, U., Hotez, P. J., \&Bottazzi, M. E. (2020). The SARS-CoV-2 vaccine pipeline: an overview. Current tropical medicine reports, 1-4.

8. Chinnaswamy, S. (2020). SARS-CoV-2 infection in India bucks the trend: Trained innate immunity? American Journal of Human Biology, e23504.

9. Chowdhury, M. A., Hossain, N., Kashem, M. A., Shahid, M. A., \& Alam, A. (2020). Immune response in COVID-19: A review. Journal of Infection and Public Health.

10. Deo, S., Manurkar, S., Krishnan, S., \& Franz, C. (2020). COVID-19 Vaccine: Development, Access and Distribution in the Indian Context. no, 378, 16.

11. Dhot, P.M., Tyagi, M., Singh, R., Bhati,P., Dhot, T.(2020). Immunization Priority of COVID-19 Vaccine in India. Int J Med Res Prof, 6(4):23-26.

12. Dubey, S., Biswas, P., Ghosh, R., Chatterjee, S., Dubey, M. J., Chatterjee, S., ... \& Lavie, C. J. (2020). Psychosocial impact of COVID-19. Diabetes \& Metabolic Syndrome: Clinical Research \& Reviews.

13. Gallo Marin, B., Aghagoli, G., Lavine, K., Yang, L., Siff, E. J., Chiang, S. S., ... \& Flanigan, T. (2020). Predictors of COVID-19 severity: A literature review. Reviews in medical virology, e2146. 
14. Giamarellos-Bourboulis, E. J., Netea, M. G., Rovina, N., Akinosoglou, K., Antoniadou, A., Antonakos, N., ... \& Ntaganou, M. (2020). Complex immune dysregulation in COVID-19 patients with severe respiratory failure. Cell host \& microbe.

15. Gorbalenya, A. E., Baker, S. C., Baric, R. S., de Groot, R. J., Drosten, C., Gulyaeva, A. A., ... \& Penzar, D. (2020). The species severe acute respiratory syndrome related coronavirus: classifying 2019-nCoV and naming it SARS-CoV-2. Nat Microbiol 5: 536-544.

16. Guan, W. J., Ni, Z. Y., Hu, Y., Liang, W. H., Ou, C. Q., He, J. X.,.. \& Du, B. (2020). Clinical characteristics of coronavirus disease 2019 in China. New England journal of medicine, 382(18), 1708-1720.

17. He, B., Wang, J., Wang, Y., Zhao, J., Huang, J., Tian, Y.,.. \& Zhou, X. (2020). The metabolic changes and immune profiles in patients with COVID-19. Frontiers in immunology, 11, 2075.

18. Huang, C., Wang, Y., Li, X., Ren, L., Zhao, J., Hu, Y., ... \& Cheng, Z. (2020). Clinical features of patients infected with 2019 novel coronavirus in Wuhan, China. The lancet, 395(10223), 497-506.

19. Huang, W., Berube, J., McNamara, M., Saksena, S., Hartman, M., Arshad, T., ... \& O'Gorman, M. (2020). Lymphocyte subset counts in COVID-19 patients: a meta-analysis. Cytometry Part A, 97(8), 772-776.

20. ICMR Serosurvey. (2020). Coronavirus: India likely had 6.4 million infections in May:ICMRSerosurvey. (https://www.thehindu.com/news/national/coronavir us-india-likely-had-64-million-infections-in-may-icmrserosurvey/article32576774.ece, accessed 29 November, 2020)

21. India Coronavirus: $9,177,840$ Cases and 134,254 Deaths - Worldometer", 2020). India Coronavirus: 9,177,840 Cases and 134,254 Deaths Worldometer. (2020). Retrieved 24 November 2020, fromhttps://www.worldometers.info/coronavirus/cou ntry/india/

22. Ji, W., Wang, W., Zhao, X., Zai, J., \& Li, X. (2020). Homologous recombination within the spike glycoprotein of the newly identified coronavirus may boost cross-species transmission from snake to human. J. Med. Virol.

23. Keni, R., Alexander, A., Nayak, P. G., Mudgal, J., \& Nandakumar, K. (2020). COVID-19: emergence, spread, possible treatments, and global burden. Frontiers in public health, 8, 216.

24. Le, T. T., Andreadakis, Z., Kumar, A., Roman, R. G., Tollefsen, S., Saville, M., \& Mayhew, S. (2020). The COVID-19 vaccine development landscape. Nat Rev Drug Discov, 19(5), 305-306.

25. Liu, Y., Yang, Y., Zhang, C., Huang, F., Wang, F., Yuan, J., ... \& Zhang, Z. (2020). Clinical and biochemical indexes from 2019-nCoV infected patients linked to viral loads and lung injury. Science China Life Sciences, 63(3), 364-374.

26. Mortaz, E., Tabarsi, P., Varahram, M., Folkerts, G., \& Adcock, I. M. (2020). The immune response and immunopathology of COVID-19. Frontiers in Immunology, 11.

27. Nisticò, V., Goeta, D., Gambini, O., \&Demartini, B. (2020). The psychological impact of COVID-19 among a sample of Italian patients with functional neurological disorders: A preliminary study. Parkinsonism \& Related Disorders, 78, 79-81

28. Ong, E. Z., Chan, Y. F. Z., Leong, W. Y., Lee, N. M. Y., Kalimuddin, S., Mohideen, S. M. H., ... \& Low, J. G. H. (2020). A dynamic immune response shapes COVID-19 progression. Cell host \& microbe.

29. Praveen, T., Desai, D., Soneja, M., \& Wig, N. (2020). Immune dysregulation in COVID-19 and its therapeutic implications. Journal of Clinical and Scientific Research, 9(1), 37.

30. Prompetchara, E., Ketloy, C., \&Palaga, T. (2020). Immune responses in COVID-19 and potential vaccines: Lessons learned from SARS and MERS epidemic. Asian Pac J Allergy Immunol, 38(1), 1-9.

31. Rao, K. S., Suryaprakash, V., Senthilkumar, R., Preethy, S., Katoh, S., Ikewaki, N., \& Abraham, S. J. (2020). Role of immune dysregulation in increased mortality among a specific subset of COVID-19 patients and immune-enhancement strategies for combatting through nutritional supplements. Frontiers in Immunology, 11, 1548.

32. Schwartz, J. L. (2020). Evaluating and Deploying Covid-19 Vaccines-The Importance of Transparency, Scientific Integrity, and Public Trust. New England Journal of Medicine.

33. Serology Survey.(2020).Banking on Serology: On Seroprevalence studies. (https://www.thehindu.com/ opinion/editorial/banking-on-serology-the-hindueditorial-on-seroprevalence-studies/article 32233451.ece, accessed 30 November, 2020)

34. Shi, Y., Wang, Y., Shao, C., Huang, J., Gan, J., Huang, X., ... \& Melino, G. (2020). COVID-19 infection: the perspectives on immune responses.

35. Singh, S., Maurya, R. P., \& Singh, R. K. (2020). "Trained immunity" from Mycobacterium spp. exposure or BCG vaccination and COVID-19 outcomes. PLoS Pathogens, 16(10), e1008969.

36. Speiser, D.E., Bachmann, M.F.(2020). COVID-19: Mechanisms of Vaccination and Immunity. Vaccines, 8(3), 404

37. Verity, R., Okell, L. C., Dorigatti, I., Winskill, P., Whittaker, C., Imai, N., ... \& Dighe, A. (2020). Estimates of the severity of coronavirus disease 2019: a model-based analysis. The Lancet infectious diseases.

38. World Health Organization. (2020). Immunization as an essential health service: guiding principles for immunization activities during the COVID-19 
pandemic and other times of severe disruption, 1 November 2020.

39. World Health Organization. (2020).Draft landscape of COVID-19 candidate vaccines. Geneva: WHO. (https://www.who.int/who-documents-detail/draftlandscape-of-covid-19-candidate-vaccines, accessed 7 December, 2020)

40. Zhang, L., Yan, X., Fan, Q., Liu, H., Liu, X., Liu, Z., \& Zhang, Z. (2020). D-dimer levels on admission to predict in-hospital mortality in patients with Covid-19. Journal of Thrombosis and Haemostasis, 18(6), 1324-1329.

41. Zhao, Y. M., Shang, Y. M., Song, W. B., Li, Q. Q., Xie, H., Xu, Q. F., ... \& Luo, H. (2020). Follow-up study of the pulmonary function and related physiological characteristics of COVID-19 survivors three months after recovery. EClinicalMedicine, 25, 100463.

42. Zheng, Z., Peng, F., Xu, B., Zhao, J., Liu, H., Peng, J., ... \& Ye, C. (2020). Risk factors of critical \& mortal COVID-19 cases: A systematic literature review and meta-analysis. Journal of Infection. 